\title{
The Modulus-Based Levenberg-Marquardt Method for Solving Linear Complementarity Problem
}

\author{
Baohua Huang and Changfeng $\mathrm{Ma}^{*}$ \\ College of Mathematics and Informatics, Fujian Key Laborotary of Mathematical \\ Analysis and Applications, Fujian Normal University, Fuzhou 350117, P. R. China
}

Received 5 November 2017; Accepted (in revised version) 23 January 2018

\begin{abstract}
As applying the Levenberg-Marquardt method to the reformulation of linear complementarity problem, a modulus-based Levenberg-Marquardt method with nonmonotone line search is established and the global convergence result is presented. Numerical experiments show that the proposed method is efficient and outperforms the modulus-based matrix splitting iteration method.
\end{abstract}

AMS subject classifications: 90C33, 65F10

Key words: Linear complementarity problem, modulus-based, Levenberg-Marquardt method, global convergence, numerical experiments.

\section{Introduction}

Let $\mathbb{R}^{n}$ and $\mathbb{R}^{n \times n}$ be the $n$-dimensional real vector space and the $n$-by- $n$ real matrix space, respectively. In this paper, we consider the linear complementarity problem, abbreviated as $\operatorname{LCP}(q, M)$, for finding a pair of real vectors $w$ and $z \in \mathbb{R}^{n}$ such that

$$
w:=M z+q \geq 0, \quad z \geq 0 \quad \text { and } \quad z^{T} w=0,
$$

where $M=\left(m_{i j}\right) \in \mathbb{R}^{n \times n}$ is a given large, sparse and real matrix, and

$$
q=\left(q_{1}, q_{2}, \cdots, q_{n}\right)^{T} \in \mathbb{R}^{n}
$$

is a given real vector. Here, the notation $\geq$ denotes the componentwise defined partial ordering between two vectors and the superscript $T$ denotes the transpose of a vector.

The linear complementarity problem was introduced by Lemke in 1964, but it was Cottle and Dantzig [1] who formally defined the linear complementarity problem and called it the fundamental problem. The $\operatorname{LCP}(q, M)$ of the form (1.1) often arises in many scientific computing and engineering applications, e.g., the linear and quadratic programming, the economies with institutional restrictions upon prices, the optimal stopping in Markov chain, and the free boundary problems; see $[2,3,5]$ for details.

${ }^{*}$ Corresponding author. Email address: macf@f jnu.edu.cn (C. F. Ma) 
For the solution of the large and sparse $\operatorname{LCP}(q, M)$, the pivot algorithms based on simplex type processes require too many pivots, destroy sparsity, have exponential computational complexity and suffer from round-off errors [10]. Therefore, iterative methods, such as projected relaxation method [11], were constructed and widely discussed. Mangasarian [12] and Ahn [13] established the convergence theory of the projected iterative method when the matrix is either symmetric or nonsymmetric.

By equivalently reformulating the $\operatorname{LCP}(q, M)$ as an implicit fixed-point equation, Van Bokhoven [6] presented a modulus iteration method, which was defined as the solution of linear equations at each iteration. Moreover, Bai [7] presented a class of modulus-based matrix splitting iteration methods which not only provided a general framework for the modified modulus method [8] and nonstationary extrapolated modulus algorithms [9], but also yielded a series of modulus-based relaxation methods which outperform the projected relaxation method as well as the modified modulus method in computing efficiency. With respect to matrix splitting method and modulus-based method, we can also refer to $[18,20-23,25,26,28-33]$ and the references therein.

As we all know, the implicit fixed-point equation which is equivalent to the $\operatorname{LCP}(q, M)$ is a absolute value equation. Iqbel et al. [14] proposed Levenberg-Marquardt method for solving absolute value equations, which is the combination of steepest descent and the Gauss-Newton methods. They proved the global convergence of new method when using the Goldstein line search. Li and Fukushima [15] presented a non-monotone line search for nonlinear equations, that is

$$
\left\|F\left(x_{k}+\alpha d_{k}\right)\right\|^{2} \leq\left(1+\eta_{k}\right)\left\|F\left(x_{k}\right)\right\|^{2}-\sigma_{1} \alpha^{2}\left\|d_{k}\right\|^{2}-\sigma_{2} \alpha^{2}\left\|F\left(x_{k}\right)\right\|^{2},
$$

where $F(x): \mathbb{R}^{n} \rightarrow \mathbb{R}^{n}$ is a continuous function, $\sigma_{1}$ and $\sigma_{2}$ are positive constants and the positive sequence $\left\{\eta_{k}\right\}$ satisfies

$$
\sum_{k=0}^{\infty} \eta_{k}<\infty
$$

It is noticeable that as $\alpha \rightarrow 0^{+}$, the left hand side of (1.2) goes to $\left\|F\left(x_{k}\right)\right\|^{2}$, while the right hand side tends to the positive constant $\left(1+\eta_{k}\right)\left\|F\left(x_{k}\right)\right\|^{2}$. Thus, (1.2) is satisfied for all sufficiently small $\alpha>0$. Hence, one can obtain $\alpha_{k}$ by means of a backtracking process. This non-monotone line search can guarantee the global convergence of the LevenbergMarquardt method [19].

Inspired by the above mentioned, we present the Levenberg-Marquardt method with a non-monotone line search for the $\operatorname{LCP}(q, M)$.

The outline of this paper is as follows. We give some basic notations, definitions and lemmas in Section 2 and establish the modulus-based Levenberg-Marquardt method for linear complementarity problem in Section 3. In Section 4, the global convergence of the modulus-based Levenberg-Marquardt method is proved. In Section 5, the numerical experiments are presented to show the effectiveness of our method. In the final section we give the concluding remarks. 


\section{Preliminaries}

We briefly introduce some necessary notations, definitions and lemmas. For a vector $x=\left(x_{1}, x_{2}, \cdots, x_{n}\right)^{T} \in \mathbb{R}^{n}$, the infinity norm of $x$ is defined as $\|x\|_{\infty}=\max _{1 \leq i \leq n}\left|x_{i}\right|$, the Euclidean norm of $\|x\|$ is defined as $\|x\|=\sqrt{x_{1}^{2}+\cdots+x_{n}^{2}}$ and $|x|=\left(\left|x_{1}\right|,\left|x_{2}\right|, \cdots,\left|x_{n}\right|\right)^{T}$ denotes the absolute value of $x$. For any $A \in \mathbb{R}^{n \times n},\|A\|$ denotes the spectral norm defined by $\|A\|=\max \left\{\|A x\|: x \in \mathbb{R}^{n},\|x\|=1\right\}$, where $\|x\|$ is the Euclidean norm.

Definition 2.1. ([4, Definition 1.4]) A vector-valued function $F: D \subseteq \mathbb{R}^{n} \rightarrow \mathbb{R}^{m}$ is called Lipschitz continuous if there exists a positive real constant $c$ such that for all vectors $x, y \in D$

$$
\|F(x)-F(y)\| \leq c\|x-y\| .
$$

We say $F$ is locally Lipschitz continuous if for every $x \in D$ there exists a neighborhood $U=$ $S(x, \delta)$ of $x$ such that $F$ restricted to $U$ is Lipschitz continuous.

Let $M=M_{1}-N_{1}=M_{2}-N_{2}$ be two splittings of the matrix $M \in \mathbb{R}^{n \times n}, \Omega$ be a positive diagonal matrix, $\gamma$ be a positive constant. By utilizing the matrix splitting and the idea of acceleration, the $\operatorname{LCP}(q, M)$ can be equivalently transformed into the system of implicit fixed-point equations [16]

$$
\left(M_{1}+\Omega\right) x=N_{1} x+\left(\Omega-M_{2}\right)|x|+N_{2}|x|-\gamma q .
$$

Moreover, with specific choices of the matrix splitting and iteration parameters, (2.1) can yield a series of accelerated modulus-based matrix splitting iteration methods. For example, let $M=D-L-U$ with $D,-L$ and $-U$ being the diagonal, the strictly lower-triangular and the strictly upper-triangular matrices of $M$, and

$$
M_{1}=\frac{1}{\alpha}(D-\beta L), \quad N_{1}=\frac{1}{\alpha}[(1-\alpha) D+(\alpha-\beta) L+\alpha U], \quad M_{2}=D-U \text { and } N_{2}=L,
$$

where $\alpha$ and $\beta$ are prescribed relaxation parameters. Then (2.1) reduces to the accelerated modulus-based accelerated overrelaxation (AMAOR) iteration method

$$
\begin{aligned}
& (D+\alpha \Omega-\beta L) x^{k+1} \\
= & {[(1-\alpha) D+(\alpha-\beta) L+\alpha U] x^{k}+\alpha(\Omega-D+U)\left|x^{k}\right|+\alpha L\left|x^{k+1}\right|-\alpha \gamma q . }
\end{aligned}
$$

It also gives the accelerated modulus-based successive overrelaxation (AMSOR) iteration method, the accelerated modulus-based Gauss-Seidel (AMGS) iteration method and the accelerated modulus-based Jacobi (AMJ) iteration method when $\alpha=\beta, \alpha=\beta=1$ and $\alpha=1, \beta=0$, respectively.

It is clear that the system of implicit fixed-point equations (2.1) is equivalent to the system of absolute value equations

$$
(M+\Omega) x+(M-\Omega)|x|+\gamma q=0 .
$$

Particularly, let $\Omega=I, \gamma=1$, we have the following lemma. 
Lemma 2.1. For the $\operatorname{LCP}(q, M)$, the following statements hold true:

(1) If $(w, z)$ is a solution of the $\operatorname{LCP}(q, M)$, then $x=(z-w) / 2$ satisfies the system of absolute value equations

$$
(M+I) x+(M-I)|x|+q=0 .
$$

(2) If $x$ satisfies the system of absolute value equations (2.3), then $z=|x|+x$ and $w=|x|-x$ is a solution of the $\operatorname{LCP}(q, M)$.

Let $F(x)$ be given by

$$
F(x)=(M+I) x+(M-I)|x|+q .
$$

Then solving $\operatorname{LCP}(q, M)$ is equivalent to solving the system of absolute value equations $F(x)=0$, where $F$ is a function from $\mathbb{R}^{n}$ into $\mathbb{R}^{n}$ as defined in (2.4). It is noticed that there is no method that gives a solution which converges very rapidly compared to existing methods because of the non-differentiability of the function $F$. Hence, Foutayeni et al. [27] constructed a sequence of smooth functions $F_{r} \in C^{\infty}$ which are uniformly convergent to the function $F$ and showed that an approximation solution of $\operatorname{LCP}(q, M)$ is obtained by solving $F_{r}(x)=0$ for $r$ is large enough. Here, the sequence of smooth functions $F_{r}: \mathbb{R}^{n} \rightarrow \mathbb{R}^{n}$ defined by

$$
F_{r}(x)=(M+I) x+(M-I)\left(x^{2}+e^{-r}\right)^{\frac{1}{2}}+q,
$$

where $r \in \mathbb{N}$ and

$$
\left(x^{2}+e^{-r}\right)^{\frac{1}{2}}:=\left(\left(x_{1}^{2}+e^{-r}\right)^{\frac{1}{2}},\left(x_{2}^{2}+e^{-r}\right)^{\frac{1}{2}}, \cdots,\left(x_{n}^{2}+e^{-r}\right)^{\frac{1}{2}}\right)^{T} \in \mathbb{R}^{n} .
$$

Specifically, Foutayeni et al. [27] derived the following results.

Lemma 2.2. ([27]) The sequence of smooth functions $\left\{F_{r}\right\}_{r \geq 1}$ converges uniformly to $F$ on $\mathbb{R}^{n}$ when $r \rightarrow+\infty$.

Lemma 2.3. ([27]) If $x_{r}^{*}$ is a solution of the equation $F_{r}(x)=0$, then $x_{r}^{*}$ is an approximation solution of the equation $F(x)=0$ for $r$ is large enough.

In the following analysis, our goal is to build a method for solving $F_{r}(x)=0$ for $r$ is large enough.

\section{Proposed method}

In this section, we suggest Levenberg-Marquardt method with non-monotone line search for the nonlinear equations (2.5) which is the reformulation of the $\operatorname{LCP}(q, M)$. Firstly, we take

$$
\Psi_{r}(x)=\frac{1}{2}\left\|F_{r}(x)\right\|^{2}
$$

as the merit function of (2.5). When solving (2.5) by Levenberg-Marquardt method, we obtain the Jacobian matrix of $F_{r}(x)$ is

$$
J^{(r)}(x)=F_{r}^{\prime}(x)=(M+I)+(M-I) D_{x, r},
$$


where

$$
D_{x, r}=\operatorname{diag}\left(d_{1}, d_{2}, \cdots, d_{n}\right), \quad d_{i}=\frac{x_{i}}{\sqrt{x_{i}^{2}+e^{-r}}} .
$$

Together with the definition of $F_{r}(x)$, we have the following lemma.

\section{Lemma 3.1.}

(1) $F_{r}(x)$ is Lipschitz continuous.

(2) $J^{(r)}(x)=F_{r}^{\prime}(x)$ is Lipschitz continuous.

(3) $J^{(r)}(x)=F_{r}^{\prime}(x)$ is bounded.

Proof. (1) Let us use the result of mean value theorem [4, Theorem 1.5] on vector function $F_{r}$. Then for all $x, y$, we have

$$
\left\|F_{r}(y)-F_{r}(x)\right\| \leq \sup _{0 \leq t \leq 1}\left\|F_{r}^{\prime}(x+t(y-x))\right\|\|y-x\| .
$$

Let $z=x+t(y-x)$. By using (3.2) and (3.3), we have

$$
\begin{aligned}
& \sup _{0 \leq t \leq 1}\left\|F_{r}^{\prime}(z)\right\|=\sup _{0 \leq t \leq 1}\left\|(M+I)+(M-I) D_{z, r}\right\| \\
= & \sup _{0 \leq t \leq 1}\left\|(M+I)+(M-I) \operatorname{diag}\left(\frac{z_{1}}{\sqrt{z_{1}^{2}+e^{-r}}}, \cdots, \frac{z_{n}}{\sqrt{z_{n}^{2}+e^{-r}}}\right)\right\| \\
\leq & \sup _{0 \leq t \leq 1}\left\{\|M+I\|+\|M-I\| \max _{1 \leq i \leq n}\left|\frac{z_{i}}{\sqrt{z_{i}^{2}+e^{-r}}}\right|\right\} \leq\|M+I\|+\|M-I\| .
\end{aligned}
$$

Together (3.4) with (3.5) yields

$$
\begin{aligned}
& \left\|F_{r}(y)-F_{r}(x)\right\| \leq \sup _{0 \leq t \leq 1}\left\|F_{r}^{\prime}(x+t(y-x))\right\|\|y-x\| \\
= & \sup _{0 \leq t \leq 1}\left\|F_{r}^{\prime}(z)\right\|\|y-x\| \leq(\|M+I\|+\|M-I\|)\|y-x\| .
\end{aligned}
$$

Hence, from Definition 2.1, $F_{r}(x)$ is Lipschitz continuous.

(2) From (3.2), by some calculations, we have

$$
\begin{aligned}
& \left\|F_{r}^{\prime}(y)-F_{r}^{\prime}(x)\right\|=\left\|\left[(M+I)+(M-I) D_{y, r}\right]-\left[(M+I)+(M-I) D_{x, r}\right]\right\| \\
= & \left\|(M-I)\left(D_{y, r}-D_{x, r}\right)\right\| \leq\|M-I\|\left\|D_{y, r}-D_{x, r}\right\| \\
= & \|M-I\| \max _{1 \leq i \leq n}\left|\frac{y_{i}}{\sqrt{y_{i}^{2}+e^{-r}}}-\frac{x_{i}}{\sqrt{x_{i}^{2}+e^{-r}}}\right| \\
= & \|M-I\| \max _{1 \leq i \leq n}\left|\frac{e^{-r}}{\left(z_{i}^{2}+e^{-r}\right)^{3 / 2}}\left(y_{i}-x_{i}\right)\right|, z_{i}=x_{i}+t\left(y_{i}-x_{i}\right), \quad t \in(0,1) \\
\leq & e^{\frac{r}{2}}\|M-I\| \max _{1 \leq i \leq n}\left|y_{i}-x_{i}\right|=e^{\frac{r}{2}}\|M-I\|\|y-x\|_{\infty} \leq e^{\frac{r}{2}}\|M-I\|\|y-x\|,
\end{aligned}
$$


where the fifth equality use the Lagrange mean value theorem of real value function $g(\xi)=$ $\frac{\xi}{\sqrt{\xi^{2}+e^{-r}}}$. It then follows from Definition 2.1 that $F_{r}^{\prime}(x)$ is Lipschitz continuous.

(3) The result can be obtained by the analysis of (1). The proof is completed.

Now, we can outline our new Levenberg-Marquardt method with non-monotone line search for solving linear complementarity problem as follows:

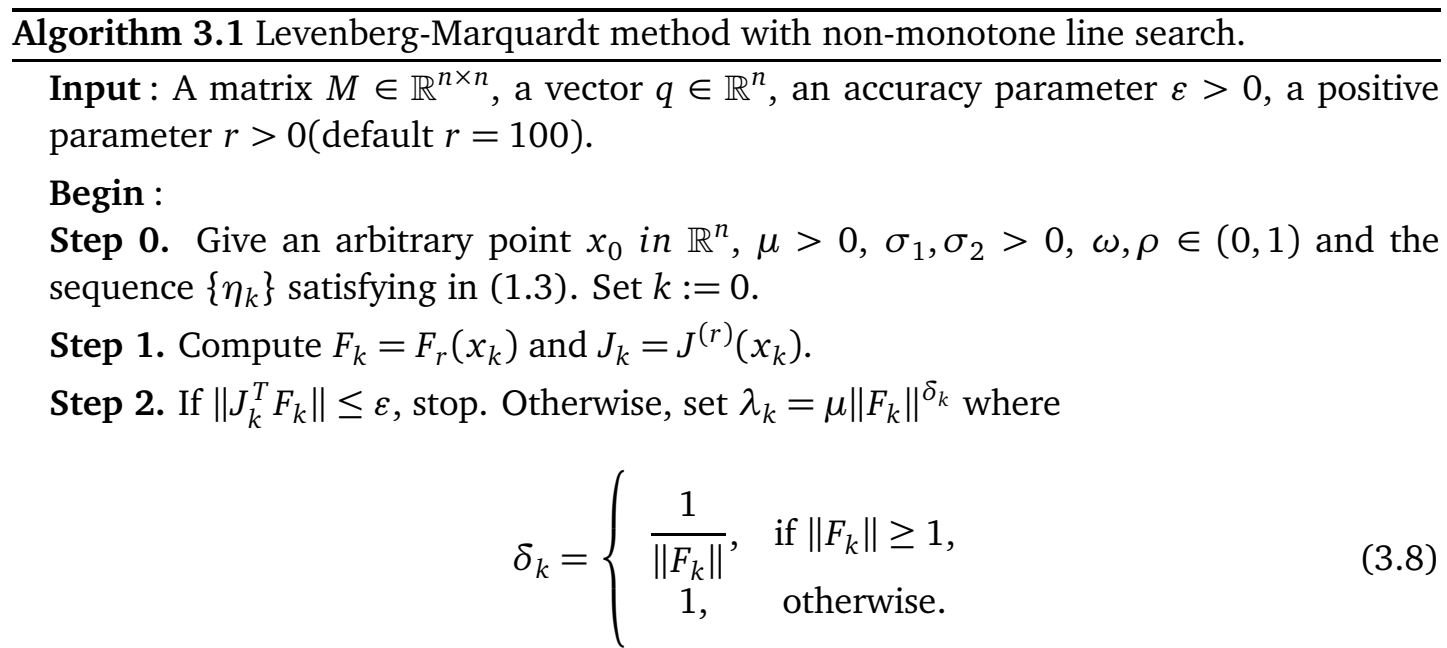

Step 3. Solve the linear system

$$
\left(J_{k}^{T} J_{k}+\lambda_{k} I\right) d=-J_{k}^{T} F_{k}
$$

to compute $d_{k}$.

Step 4. If

$$
\left\|F_{r}\left(x_{k}+d_{k}\right)\right\| \leq \omega d_{k},
$$

then, take $\alpha_{k}=1$ and go to Step 6. Otherwise, go to Step 5 .

Step 5. Compute $\alpha_{k}=\max \left\{1, \rho^{1}, \rho^{2}, \cdots\right\}$ with $\alpha_{k}=\rho^{i}$ satisfying

$$
\left\|F_{r}\left(x_{k}+\alpha_{k} d_{k}\right)\right\|^{2} \leq\left(1+\eta_{k}\right)\left\|F_{r}\left(x_{k}\right)\right\|^{2}-\sigma_{1} \alpha_{k}^{2}\left\|d_{k}\right\|^{2}-\sigma_{2} \alpha_{k}^{2}\left\|F_{r}\left(x_{k}\right)\right\|^{2},
$$

where the positive sequence $\left\{\eta_{k}\right\}$ satisfies (1.3).

Step 6. Set $x_{k+1}=x_{k}+\alpha_{k} d_{k}$. Set $k:=k+1$ and go to Step 1 .

end

Remark 3.1. As $\alpha \rightarrow 0^{+}$, the left hand side of (3.11) goes to $\left\|F_{k}\right\|^{2}$ while the right hand side tends to the positive value $\left(1+\eta_{k}\right)\left\|F_{k}\right\|^{2}$, thus (3.11) is satisfied for sufficiently small $\alpha>0$. This shows that Algorithm 3.1 is efficient. 


\section{Convergence analysis}

In this section, we will show that Algorithms 3.1 is global convergence. Firstly, we define

$$
\Omega=\left\{x \mid\left\|F_{r}(x)\right\| \leq e^{\eta / 2}\left\|F_{0}\right\|\right\},
$$

where $\eta$ is a positive constant such that

$$
\sum_{k=0}^{\infty} \eta_{k} \leq \eta<\infty
$$

According to Lemma 3.1, $F_{r}(x)$ is Lipschitz continuous and its Jacobian matrix $J^{(r)}(x)$ is Lipschitz continuous and bounded, so $F_{r}(x)$ and $J^{(r)}(x)$ have the same properties on $\Omega$, i.e., there exists a positive constant $L$ such that for all $x, y \in \Omega$

$$
\begin{aligned}
& \|F(x)-F(y)\| \leq L\|x-y\|, \\
& \left\|J^{(r)}(x)-J^{(r)}(y)\right\| \leq L\|x-y\|, \\
& \left\|J^{(r)}(x)\right\| \leq L .
\end{aligned}
$$

Now we state the following two lemmas that show the sequence $\left\{x_{k}\right\}$ generated by Algorithm 3.1 belongs to $\Omega$ and the sequence $\left\{\left\|F_{k}\right\|\right\}$ converges.

Lemma 4.1. ([17])Let $\left\{a_{k}\right\}$ and $\left\{r_{k}\right\}$ be positive sequences satisfying $a_{k+1} \leq\left(1+r_{k}\right) a_{k}+r_{k}$, $\forall k=0,1, \cdots$ and $\sum_{k=0}^{\infty} r_{k}<\infty$. Then $\left\{a_{k}\right\}$ converges.

Lemma 4.2. Let the sequence $\left\{x_{k}\right\}$ be generated by Algorithm 3.1. Then

(1) the sequence $\left\{\left\|F_{k}\right\|\right\}$ converges and $x_{k} \in \Omega$ for all $k \geq 0$.

(2) the sequence $\left\{\left\|F_{k}\right\|\right\}$ is bounded, that is, there exists a constant $\mathscr{M}>0$ such that

$$
\left\|F_{k}\right\| \leq \mathscr{M}, \quad \forall k \geq 0 .
$$

Proof. From (3.10) and (3.11), we have

$$
\left\|F_{k+1}\right\|^{2} \leq\left(1+\eta_{k}\right)\left\|F_{k}\right\|^{2} .
$$

Due to $\left\{\eta_{k}\right\}$ is a positive sequence, we have

$$
\left\|F_{k+1}\right\|^{2} \leq\left(1+\eta_{k}\right)\left\|F_{k}\right\|^{2}+\eta_{k} .
$$

Lemma 4.1 implies that $\left\{\left\|F_{k}\right\|^{2}\right\}$ and so $\left\{\left\|F_{k}\right\|\right\}$ are convergent. Moreover, from the above inequality, we deduce that

$$
\left\|F_{k+1}\right\| \leq\left(1+\eta_{k}\right)^{1 / 2}\left\|F_{k}\right\| \leq \cdots \leq \prod_{i=0}^{k}\left(1+\eta_{i}\right)^{1 / 2}\left\|F_{0}\right\| .
$$


Thus

$$
\begin{aligned}
\left\|F_{k+1}\right\| & \leq\left(\frac{1}{k+1} \sum_{i=0}^{k}\left(1+\eta_{i}\right)\right)^{\frac{k+1}{2}}\left\|F_{0}\right\| \leq\left(1+\sum_{i=0}^{k} \eta_{i} /(k+1)\right)^{\frac{k+1}{2}}\left\|F_{0}\right\| \\
& \leq\left(1+\frac{\eta}{k+1}\right)^{\frac{k+1}{2}}\left\|F_{0}\right\| \leq e^{\eta / 2}\left\|F_{0}\right\|,
\end{aligned}
$$

where the second inequality use arithmetic-geometric means inequality, the third inequality use the basis fact $\lim _{n \rightarrow \infty}(1+1 / n)^{n}=e$ and the relation (4.2). This inequality means $x_{k} \in \Omega$ for all $k$. The proof of (1) is completed. Part (1) and the definition of $\Omega$ implies that the sequence $\left\{\left\|F_{k}\right\|\right\}$ is bounded. The proof is completed.

Lemma 4.3. Let the sequence $\left\{x_{k}\right\}$ be generated by Algorithm 3.1. If (3.10) holds for infinite $k$, then $\left\{\left\|F_{k}\right\|\right\}$ converges to zero. In other words, if there exists a positive constant $c$ such that $\left\|F_{k}\right\| \geq c$ holds for sufficiently large $k$, then (3.10) holds for finite $k$.

Proof. Denote the index sets

$$
I_{j}=\{k \leq j \mid(3.10) h o l d s\}, \quad H_{j}=\{0,1, \cdots, j\} \backslash I_{j}, \quad j=1,2, \cdots .
$$

If (3.10) holds for infinite $k$, then as $j \rightarrow \infty, \operatorname{card}\left(I_{j}\right) \rightarrow \infty$, where $\operatorname{card}\left(I_{j}\right)$ is the number elements of $I_{j}$. From (3.10) and (3.11), we have

$$
\begin{aligned}
\left\|F_{k+1}\right\| & \leq\left(\prod_{i \in H_{k}}\left(1+\eta_{i}\right)^{1 / 2} \prod_{i \in I_{k}} \eta\right)\left\|F_{0}\right\| \\
& =\left(\prod_{i \in H_{k}}\left(1+\eta_{i}\right)^{1 / 2}\right) \rho^{\operatorname{card}\left(I_{k}\right)}\left\|F_{0}\right\| \\
& \leq e^{\frac{\eta}{2}} \rho^{\operatorname{card}\left(I_{k}\right)}\left\|F_{0}\right\| \rightarrow 0, \text { as } k \rightarrow \infty .
\end{aligned}
$$

So $\left\|F_{k}\right\| \rightarrow 0$. The proof is completed.

Theorem 4.1. Algorithm 3.1 either terminates in a finite number of steps or satisfies

$$
\liminf _{k \rightarrow \infty}\left\|J_{k}^{T} F_{k}\right\|=0 \text {. }
$$

Proof. By contradiction, suppose there exist $\tau>0$ and an integer $\bar{k}$ such that

$$
\left\|J_{k}^{T} F_{k}\right\| \geq \tau, \quad \forall k \geq \bar{k}
$$

This together with (4.3c) implies that

$$
\left\|F_{k}\right\| \geq L^{-1} \tau
$$

holds for sufficiently large $k$. So, by Lemma 4.3, the inequality (3.10) holds for finite $k$. On the other hand, from (3.11), we have

$$
\left\|F_{k+1}\right\|^{2} \leq\left(1+\eta_{k}\right)\left\|F_{k}\right\|^{2}-\sigma_{2} \alpha_{k}^{2}\left\|F_{k}\right\|^{2},
$$


then

$$
\sigma_{2} \alpha_{k}^{2}\left\|F_{k}\right\|^{2} \leq\left\|F_{k}\right\|^{2}-\left\|F_{k+1}\right\|^{2}+\eta_{k}\left\|F_{k}\right\|^{2} .
$$

Thus, according to Lemma 4.2 (2), results that

$$
\sigma_{2} \sum_{k=0}^{m} \alpha_{k}^{2}\left\|F_{k}\right\|^{2} \leq\left\|F_{0}\right\|^{2}-\left\|F_{m+1}\right\|^{2}+\sum_{k=0}^{m} \eta_{k}\left\|F_{k}\right\|^{2} \leq\left\|F_{0}\right\|^{2}+M \sum_{k=0}^{m} \eta_{k},
$$

which implies

$$
\sum_{k=0}^{\infty} \alpha_{k}^{2}\left\|F_{k}\right\|^{2}<\infty
$$

Then $\lim _{k \rightarrow \infty} \alpha_{k}\left\|F_{k}\right\|=0$. This relation together with (4.7) yields

$$
\lim _{k \rightarrow \infty} \alpha_{k}=0
$$

Now, let $J_{k}=U_{k} \Sigma_{k} V_{k}^{T}$ be the sigular value decomposition (SVD) of $J_{k}$, where $U_{k}, V_{k}$ are two orthogonal matrices and $\Sigma_{k}=\operatorname{diag}\left(\sigma_{k, 1}, \sigma_{k, 2}, \cdots, \sigma_{k, n}\right)$ with $\sigma_{k, 1} \geq \sigma_{k, 2} \geq \cdots \sigma_{k, n} \geq$ 0 . Then, we have

$$
\begin{aligned}
& \left\|\left(J_{k}^{T} J_{k}+\lambda_{k} I\right)^{-1}\right\|=\left\|V_{k}\left(\Sigma_{k}^{2}+\lambda_{k} I\right)^{-1} V_{k}^{T}\right\| \\
= & \left\|\left(\Sigma_{k}^{2}+\lambda_{k} I\right)^{-1}\right\|=\max _{i \in\{1,2, \cdots, n\}}\left(\sigma_{k, i}^{2}+\lambda_{k} I\right)^{-1} \leq \lambda_{k}^{-1} .
\end{aligned}
$$

This inequality together with (3.9), (4.3c) and (4.4) implies that

$$
\begin{aligned}
\left\|d_{k}\right\| & =\left\|\left(J_{k}^{T} J_{k}+\lambda_{k} I\right)^{-1} J_{k}^{T} F_{k}\right\| \leq\left\|\left(J_{k}^{T} J_{k}+\lambda_{k} I\right)^{-1}\right\|\left\|J_{k}\right\|\left\|F_{k}\right\| \\
& \leq L \lambda_{k}^{-1}\left\|F_{k}\right\|=\frac{L}{\mu}\left\|F_{k}\right\|^{1-\delta_{k}} .
\end{aligned}
$$

If $\left\|F_{k}\right\|<1$, then $\delta_{k}=1$, hence

$$
\left\|d_{k}\right\| \leq \frac{L}{\mu}
$$

If $\left\|F_{k}\right\| \geq 1$, then $\delta_{k}=\frac{1}{\left\|F_{k}\right\|}$, hence

$$
\left\|d_{k}\right\| \leq \frac{L}{\mu}\left\|F_{k}\right\|^{1-\frac{1}{\left\|F_{k}\right\|}} \leq \frac{L}{\mu}\left\|F_{k}\right\| \leq \frac{L}{\mu} \mathscr{M} .
$$

Let $\mathscr{M}_{1}=\max \{1, \mathscr{M}\}$, together (4.10) with (4.11) yields

$$
\left\|d_{k}\right\| \leq \frac{L}{\mu} \mathscr{M}_{1} .
$$

If $\liminf _{k \rightarrow \infty}\left\|d_{k}\right\|=0$, then we have from (3.9) and (4.3c) that

$$
\liminf _{k \rightarrow \infty}\left\|J_{k}^{T} F_{k}\right\|=\liminf _{k \rightarrow \infty}\left\|\left(J_{k}^{T} J_{k}+\lambda_{k} I\right) d_{k}\right\|=0
$$


which obtains a contradiction to (4.6). Hence there exists a constant $v>0$, such that

$$
\liminf _{k \rightarrow \infty}\left\|d_{k}\right\|>v
$$

From line search (3.11), we have

$$
\begin{gathered}
\left\|F\left(x_{k}+\bar{\alpha}_{k} d_{k}\right)\right\|^{2}-\left\|F_{k}\right\|^{2} \\
>-\bar{\alpha}_{k}^{2}\left(\sigma_{1}\left\|d_{k}\right\|^{2}+\sigma_{2}\left\|F_{k}\right\|^{2}\right)+\eta_{k}\left\|F_{k}\right\|^{2}>-\bar{\alpha}_{k}^{2}\left(\sigma_{1}\left\|d_{k}\right\|^{2}+\sigma_{2}\left\|F_{k}\right\|^{2}\right),
\end{gathered}
$$

where $\bar{\alpha}_{k}=\frac{\alpha_{k}}{\rho}$. Combine this inequality with (4.3) yields

$$
\begin{aligned}
& \bar{\alpha}_{k}^{2}\left(\sigma_{1}\left\|d_{k}\right\|^{2}+\sigma_{2}\left\|F_{k}\right\|^{2}\right)>-\left(\left\|F\left(x_{k}+\bar{\alpha}_{k} d_{k}\right)\right\|^{2}-\left\|F_{k}\right\|^{2}\right) \\
= & -2 F_{k}^{T}\left[F\left(x_{k}+\bar{\alpha}_{k} d_{k}\right)-F_{k}\right]-\left\|F\left(x_{k}+\bar{\alpha}_{k} d_{k}\right)-F_{k}\right\|^{2} \\
\geq & -2 F_{k}^{T}\left[F\left(x_{k}+\bar{\alpha}_{k} d_{k}\right)-F_{k}\right]-L^{2} \bar{\alpha}_{k}^{2}\left\|d_{k}\right\|^{2} .
\end{aligned}
$$

On the other hand, by the mean-value theorem [4, Theorem 1.6], we have

$$
\begin{aligned}
& F_{k}^{T}\left[F\left(x_{k}+\bar{\alpha}_{k} d_{k}\right)-F_{k}\right]=\bar{\alpha}_{k} F_{k}^{T} J_{k} d_{k}+F_{k}^{T} \int_{0}^{1}\left(J\left(x_{k}+t \bar{\alpha}_{k} d_{k}\right)-J_{k}\right) \bar{\alpha}_{k} d_{k} d t \\
\leq & \bar{\alpha}_{k} F_{k}^{T} J_{k} d_{k}+\frac{1}{2} L \mathscr{M} \bar{\alpha}_{k}^{2}\left\|d_{k}\right\|^{2}=-\bar{\alpha}_{k} d_{k}^{T}\left(J_{k}^{T} J_{k}+\lambda_{k} I\right) d_{k}+\frac{1}{2} L \mathscr{M} \bar{\alpha}_{k}^{2}\left\|d_{k}\right\|^{2},
\end{aligned}
$$

which, together with (4.15), yields

$$
\bar{\alpha}_{k}\left(\sigma_{1}\left\|d_{k}\right\|^{2}+\sigma_{2}\left\|F_{k}\right\|^{2}\right)>2 d_{k}^{T}\left(J_{k}^{T} J_{k}+\lambda_{k} I\right) d_{k}-L \mathscr{M} \bar{\alpha}_{k}\left\|d_{k}\right\|^{2}-L^{2} \bar{\alpha}_{k}\left\|d_{k}\right\|^{2} .
$$

Hence

$$
\bar{\alpha}_{k}\left[\left(\sigma_{1}+L \mathscr{M}+L^{2}\right)\left\|d_{k}\right\|^{2}+\sigma_{2}\left\|F_{k}\right\|^{2}\right]>2 d_{k}^{T}\left(J_{k}^{T} J_{k}+\lambda_{k} I\right) d_{k} \geq 2 \lambda_{k}\left\|d_{k}\right\|^{2},
$$

where the last inequality is due to the semi-positive definite of $J_{k}^{T} J_{k}$. So, from the inequality (4.17), we have

$$
\bar{\alpha}_{k}>\frac{2 \lambda_{k}\left\|d_{k}\right\|^{2}}{\left(\sigma_{1}+L \mathscr{M}+L^{2}\right)\left\|d_{k}\right\|^{2}+\sigma_{2}\left\|F_{k}\right\|^{2}} .
$$

Consequently, we can deduce from (4.4), (4.12), (4.14) and (4.18) that $\left\{\alpha_{k}\right\}$ is bounded away from zero, which contradicts with (4.8) and the proof is completed.

\section{Numerical experiments}

In this section, we represent some numerical examples to demonstrate the effectiveness of our algorithm from the aspects of iteration steps (denoted by 'Iter'), elapsed CPU time in 
seconds (denoted by 'CPU') and the norm of absolute residual vectors (denoted by 'Res'). Here, 'Res' is defined as

$$
\operatorname{Res}\left(z^{k}\right):=\left\|\min \left(M z^{k}+q, z^{k}\right)\right\|_{2}
$$

where $z^{k}$ is the $k$ th approximate solution to the linear complementarity problem (1.1), and the minimum is taken componentwise.

All of the tests were run on the Intel (R) Core (TM), where the CPU is $2.40 \mathrm{GHz}$ and the memory is $8.0 \mathrm{~GB}$, the programming language was MATLAB R2015a. The stopping criteria for all methods are $\operatorname{Res}\left(z^{k}\right) \leq 10^{-5}$ or $k$ reaches the maximal number of iteration, e.g., 5000. Moreover, for Algorithm 3.1, we set the parameter $r=100, \mu=0.5, \sigma_{1}=$ $\sigma_{2}=0.55, \omega=0.5, \rho=0.8, \eta_{k}=0.5^{k}$.

We compare our method with 'AMSOR' method presented in [16] as follows:

$$
\begin{aligned}
& (D+\alpha \Omega-\alpha L) x^{k+1} \\
= & {[(1-\alpha) D+\alpha U] x^{k}+\alpha(\Omega-D+U)\left|x^{k}\right|+\alpha L\left|x^{k+1}\right|-\alpha \gamma q . }
\end{aligned}
$$

In numerical experiments, take $\gamma=1$ and $\Omega=5 D$, and have 'AMSOR' converges, we also take different $\alpha$ for comparison.

Example 5.1. ([16]) Let $m$ be a prescribed positive integer and $n=m^{2}$. Consider the $\operatorname{LCP}(q, M)$, in which $M \in \mathbb{R}^{n \times n}$ is given by $M=\widehat{M}+v I$ and $q \in \mathbb{R}^{n}$ is given by $q=$ $-\left(\frac{1}{\alpha} D-L\right) z^{*}$, where

$$
\widehat{M}=\left(\begin{array}{cccccc}
B & -I & O & \cdots & O & O \\
-I & B & -I & \cdots & O & O \\
O & -I & B & \cdots & O & O \\
\vdots & \vdots & & \ddots & \vdots & \vdots \\
O & O & \cdots & \cdots & B & -I \\
O & O & \cdots & \cdots & -I & B
\end{array}\right), \quad z^{*}=\left(\begin{array}{c}
1 \\
2 \\
1 \\
2 \\
\vdots
\end{array}\right)
$$

where $B=\operatorname{tridiag}(-1,4,-1) \in \mathbb{R}^{m \times m}, I \in \mathbb{R}^{m \times m}$ is a unit matrix, $O \in \mathbb{R}^{m \times m}$ is the zero matrix, $v$ is a constant and $\alpha$ is the positive parameter used in 'AMSOR'. Obviously, $M$ is a symmetric positive definite matrix. In this example, we take $v=4$.

In Table 1, the iteration steps, the CPU time and the residual norms for the LevenbergMarquardt method (Algorithm 3.1) and the accelerated modulus-based matrix splitting iteration method (AMSOR) for Example 5.1 are listed.

From Table 1, we can find that Algorithm 3.1 has higher precision for different choices $n$. Algorithm 3.1 needs less CPU time and iteration number. Especially, the iteration number of Algorithm 3.1 is far less than that of 'AMSOR' method. 
Table 1: Numerical comparison of the testing methods for Example 5.1.

\begin{tabular}{||c|c|cccc|cccc||}
\hline \multirow{2}{*}{$\mathrm{m}$} & & \multicolumn{5}{|c|}{ AMSOR([16]) } & \multicolumn{4}{c||}{ Algorithm 3.1 } \\
\cline { 3 - 10 } & & $(\alpha=0.8)$ & $(\alpha=0.9)$ & $(\alpha=1.1)$ & $(\alpha=1.2)$ & $(\alpha=0.8)$ & $(\alpha=0.9)$ & $(\alpha=1.1)$ & $(\alpha=1.2)$ \\
\hline \multirow{3}{*}{10} & Iter & 90 & 74 & 57 & 54 & 3 & 3 & 3 & 3 \\
& CPU & 0.0577 & 0.0564 & 0.0434 & 0.0293 & 0.0280 & 0.0299 & 0.0291 & 0.0282 \\
& Res & $9.8518 \mathrm{e}-06$ & $9.8531 \mathrm{e}-06$ & $9.5125 \mathrm{e}-06$ & $7.6435 \mathrm{e}-06$ & $2.6153 \mathrm{e}-08$ & $8.4000 \mathrm{e}-09$ & $1.7749 \mathrm{e}-09$ & $5.3528 \mathrm{e}-10$ \\
\hline \multirow{3}{*}{20} & Iter & 98 & 81 & 61 & 57 & 3 & 3 & 3 & 3 \\
& CPU & 0.2652 & 0.2961 & 0.2122 & 0.1594 & 0.1403 & 0.1387 & 0.1433 & 0.1319 \\
& Res & $9.2125 \mathrm{e}-06$ & $9.3818 \mathrm{e}-06$ & $9.6478 \mathrm{e}-06$ & $9.9704 \mathrm{e}-06$ & $6.4034 \mathrm{e}-07$ & $1.9456 \mathrm{e}-07$ & $2.5338 \mathrm{e}-08$ & $8.0523 \mathrm{e}-09$ \\
\hline \multirow{3}{*}{30} & Iter & 101 & 84 & 63 & 59 & 4 & 3 & 3 & 3 \\
& CPU & 1.1397 & 1.0281 & 0.7868 & 0.7513 & 0.9609 & 0.7050 & 0.7298 & 0.7342 \\
& Res & $9.8448 \mathrm{e}-06$ & $9.3716 \mathrm{e}-06$ & $9.7976 \mathrm{e}-06$ & $9.8046 \mathrm{e}-06$ & $7.9535 \mathrm{e}-14$ & $1.0505 \mathrm{e}-06$ & $1.7578 \mathrm{e}-07$ & $5.0147 \mathrm{e}-08$ \\
\hline \multirow{3}{*}{40} & Iter & 104 & 86 & 65 & 61 & 4 & 4 & 3 & 3 \\
& CPU & 4.4286 & 3.6525 & 2.7851 & 2.4528 & 3.7787 & 3.3502 & 2.6663 & 2.5685 \\
& Res & $8.6993 \mathrm{e}-06$ & $9.2668 \mathrm{e}-06$ & $8.5050 \mathrm{e}-06$ & $8.1384 \mathrm{e}-06$ & $1.7006 \mathrm{e}-13$ & $9.0236 \mathrm{e}-14$ & $4.9956 \mathrm{e}-07$ & $1.7792 \mathrm{e}-07$ \\
\hline \multirow{3}{*}{50} & Iter & 105 & 88 & 66 & 62 & 4 & 4 & 3 & 3 \\
& CPU & 12.0005 & 10.0970 & 7.6646 & 7.1613 & 10.8706 & 10.0492 & 7.8830 & 7.7892 \\
& Res & $9.6343 \mathrm{e}-06$ & $8.3616 \mathrm{e}-06$ & $8.6148 \mathrm{e}-06$ & $8.0671 \mathrm{e}-06$ & $4.1890 \mathrm{e}-13$ & $1.6673 \mathrm{e}-13$ & $1.0589 \mathrm{e}-06$ & $3.6835 \mathrm{e}-07$ \\
\hline
\end{tabular}

Example 5.2. ([24])Consider the $\operatorname{LCP}(q, M), M \in \mathbb{R}^{n \times n}$ and $q \in \mathbb{R}^{n}$ are given below:

$$
M=\left(\begin{array}{cccccc}
4 & -2 & 0 & \cdots & 0 & 0 \\
1 & 4 & -2 & \cdots & 0 & 0 \\
0 & 1 & 4 & \cdots & 0 & 0 \\
\vdots & \vdots & \vdots & \ddots & \vdots & \vdots \\
0 & 0 & 0 & \cdots & 4 & -2 \\
0 & 0 & 0 & \cdots & 1 & 4
\end{array}\right), \quad q=\left(\begin{array}{l}
-4 \\
-4 \\
-4 \\
\vdots \\
-4 \\
-4
\end{array}\right) \in \mathbb{R}^{n} .
$$

It is easy to see that $M$ is a nonsymmetric tridiagonal $H_{+}$-matrix.

In table 2, the iteration steps, the CPU time and the residual norms for the LevenbergMarquardt method (Algorithm 3.1) and the accelerated modulus-based matrix splitting iteration method (AMSOR) for Example 5.2 are listed.

From Table 2, we can find that Algorithm 3.1 has higher precision for different choices $n$. When $\alpha=0.8$ and $\alpha=0.9$, the CPU time and the iteration number of Algorithm 3.1 are far less than that of 'AMSOR' method. When $\alpha=1.1$ and $\alpha=1.2$, with the increasing of matrix dimension $n$, Algorithm 3.1 needs more CPU time. However, Algorithm 3.1 outperforms 'AMSOR' method in terms of the iteration number and the precision.

\section{Conclusions}

In this paper, the modulus-based Levenberg-Marquardt method is proposed and applied to the linear complementarity problem. The proposed method is well defined, the new algorithm is globally convergent by utilizing the non-monotone line search. Numerical 
Table 2: Numerical comparison of the testing methods for Example 5.2.

\begin{tabular}{||c|cccccc||}
\hline \multirow{2}{*}{$\operatorname{Dim}(\mathrm{n})$} & & $\begin{array}{c}\text { AMSOR([16]) } \\
(\alpha=0.8)\end{array}$ & $\begin{array}{c}\text { AMSOR([16]) } \\
(\alpha=0.9)\end{array}$ & $\begin{array}{c}\text { AMSOR([16]) } \\
(\alpha=1.1)\end{array}$ & $\begin{array}{c}\text { AMSOR([16]) } \\
(\alpha=1.2)\end{array}$ & Algorithm 3.1 \\
\hline \multirow{3}{*}{100} & Iter & 70 & 62 & 50 & 46 & 3 \\
& CPU & 0.0430 & 0.0393 & 0.0383 & 0.0405 & 0.0261 \\
& Res & $9.1563 \mathrm{e}-06$ & $8.9179 \mathrm{e}-06$ & $9.3475 \mathrm{e}-06$ & $8.1329 \mathrm{e}-06$ & $2.6713 \mathrm{e}-08$ \\
\hline \multirow{4}{*}{400} & Iter & 74 & 65 & 53 & 48 & 3 \\
& CPU & 0.2354 & 0.1972 & 0.1611 & 0.1635 & 0.1128 \\
& Res & $8.3568 \mathrm{e}-06$ & $9.2419 \mathrm{e}-06$ & $8.2115 \mathrm{e}-06$ & $9.0419 \mathrm{e}-06$ & $3.4113 \mathrm{e}-07$ \\
\hline \multirow{3}{*}{900} & Iter & 76 & 67 & 54 & 49 & 3 \\
& CPU & 0.9845 & 0.8334 & 0.7088 & 0.6231 & 0.6050 \\
& Res & $8.3474 \mathrm{e}-06$ & $8.7428 \mathrm{e}-06$ & $9.2991 \mathrm{e}-06$ & $9.9547 \mathrm{e}-06$ & $1.5609 \mathrm{e}-06$ \\
\hline \multirow{3}{*}{1500} & Iter & 77 & 68 & 55 & 50 & 4 \\
& CPU & 2.7097 & 2.4697 & 2.0325 & 1.8528 & 2.5815 \\
& Res & $8.7810 \mathrm{e}-06$ & $8.9502 \mathrm{e}-06$ & $9.0071 \mathrm{e}-06$ & $9.3739 \mathrm{e}-06$ & $2.3943 \mathrm{e}-13$ \\
\hline \multirow{3}{*}{2000} & Iter & 78 & 69 & 56 & 51 & 4 \\
& CPU & 5.3375 & 5.2777 & 4.0042 & 3.4825 & 5.1278 \\
& Res & $8.2470 \mathrm{e}-06$ & $8.1804 \mathrm{e}-06$ & $7.7889 \mathrm{e}-06$ & $7.8805 \mathrm{e}-06$ & $5.5586 \mathrm{e}-13$ \\
\hline
\end{tabular}

results indicate that the modulus-based Levenberg-Marquardt method with non-monotone line search is effective and robust for solving linear complementarity problem. Moreover, the modulus-based Levenberg-Marquardt method outperforms 'AMSOR' method in terms of the iteration number, the precision and the CPU time.

Acknowledgments The authors are grateful to the anonymous referees for their useful suggestions and comments that improved the presentation of this paper. This research is supported by National Science Foundation of China (41725017), National Basic Research Program of China under grant number 2014CB845906. It is also partially supported by the CAS/CAFEA international partnership Program for creative research teams (No. KZZDEW-TZ-19 and KZZD-EW-TZ-15) and Strategic Priority Research Program of the Chinese Academy of Sciences (No. XDB18010202).

\section{References}

[1] R. W. Cottle and G. B. Dantzig, Complementary pivot theory of mathematical programming, Linear Algebra Appl., 1 (1968), pp. 103-125.

[2] R. W. Cottle, J. S. PAng And R. E. Stone, The Linear Complementarity Problem, Academic, SanDiego, 1992.

[3] K. G. Murty, Linear Complementarity, Linear and Nonlinear Programming, Heldermann, Berlin, 1988.

[4] X. D. Huang, Z. G. Zeng and Y. N. MA, The Theory and Methods for Nonlinear Numerical Analysis, Wuhan university press, Wuhan, 2004.

[5] U. SchäfER, A linear complementarity problem with a P-matrix, SIAM Rev. 46 (2004), pp. 189201. 
[6] D. Leenaerts and W. M. G. van Bokhoven, Piecewise-Linear Modelling and Analysis, Proefschrift, Eindhoven, 1981.

[7] Z. Z. BAI, Modulus-based matrix splitting iteration methods for linear complementarity problems, Numer. Linear Algebra Appl., 17 (2010), pp. 917-933.

[8] J. L. Dong AND M. Q. JIANG, A modified modulus method for symmetric positive-definite linear complementarity problems, Numer. Linear Algebra Appl., 16 (2009), pp. 129-143.

[9] A. Hadjidimos AND M. Tzoumas, Nonstationary extrapolated modulus algorithms for the solution of the linear complementarity problem, Linear Algebra Appl., 431 (2009), pp. 197-210.

[10] N. W. KAPPEL AND L. T. WATSON, Iterative algorithms for the linear complementarity problems, Int. J. Comput. Math., 19 (1986), pp. 273-297.

[11] C. W. CRYER, The solution of a quadratic programming using systematic overrelaxation, SIAM J. Control., 9 (1971), pp. 385-392 .

[12] O. L. MAngasarian, Solutions of symmetric linear complementarity problems by iterative methods, J. Comput. Theory Appl., 22 (1977), pp. 465-485.

[13] B. H. AHN, Solutions of nonsymmetric linear complementarity problems by iterative methods, J. Comput. Theory Appl., 33 (1981), pp. 175-185.

[14] J. Iqbal, A. IQbal AND M. ARIF, Levenberg-Marquardt method for solving systems of absolute value equations, J. Comput. Appl. Math., 282 (2015), pp. 134-138.

[15] D. Li And M. Funushima, A globally and superlinearly convergent Gauss-Newton-based BFGS method for symmetric nonlinear equations, SIAM J. Numer. Anal., 37 (1999), pp. 152-172 .

[16] N. ZHENG AND J. F. Yin, Accelerated modulus-based matrix splitting iterations for linear complementarity problems, Numer. Algorithms., 57 (2011), pp. 83-99.

[17] A. Berman and R. J. Plemmons, Nonnegative Matrix in the Mathematical Sciences, SIAM Publisher, Philadelphia, 1994.

[18] H. Zheng AND W. Li, The modulus-based nonsmooth Newton's method for solving linear complementarity problems, J. Comput. Appl. Math., 288 (2015), pp. 116-126.

[19] W. Y. Sun, J. Y. HAN AND J. Sun, On the global convergence of nonmonotone descent methods, J. Comput. Appl. Math., 146 (2002), pp. 89-98.

[20] Z. Z. BAI AND D. Evans, Matrix multisplitting methods with applications to linear complementarity problems: parallel synchronous and chaotic methods, Reseaux et Systemes Repartis: Calculateurs Paralleles, 13 (2001), pp. 125-154.

[21] Z. Z. BAI AND D. EvAns, Matrix multisplitting methods with applications to linear complementarity problems: parallel asynchronous methods, Int. J. Comput. Math., 79 (2002), pp. 205-232.

[22] Z. Z. BAI AND L. L. ZHANG, Modulus-based synchronous multisplitting iteration methods for linear complementarity problems, Numer. Linear Algebra Appl., 20 (2013), pp. 425-439.

[23] Z. Z. BAI AND L. L. ZHANG, Modulus-based synchronous multisplitting iteration methods for linear complementarity problems, Numer. Algorithms, 62 (2013), pp. 59-77.

[24] B. H. AHN, Iterative methods for linear complementarity problems with upper-bounds on primary variables, Math. Program, 26 (1983), pp. 295-315.

[25] J. T. Hong AND C. L. LI, Modulus-based matrix splitting iteration methods for a class of implicit complementarity problems, Numer. Linear Algebra Appl., 23 (2016), pp. 629-641.

[26] C. F. Ma And N. HuAng, Modified modulus-based matrix splitting algorithms for a class of weakly nondifferentiable nonlinear complementerity problems, Appl. Numer. Math., 108 (2016), pp. 116-124.

[27] Y. E. L. Foutayeni, H. E. L. Bouanani and M. Khalada, An $(m+1)$-step iterative method of convergence order $(m+2)$ for linear complemenarity problems, J. Appl. Math. Comput., 54 (2017), pp. 229-242.

[28] W. W. XU AND H. LIU, A modified general modulus-based matrix splitting method for linear 
complementarity problems of H-matrices, Linear Algebra. Appl., 458 (2014), pp. 626-637.

[29] W. W. Xu, Modified modulus-based matrix splitting iteration methods for linear complementarity problems, Numer. Linear Algebra. Appl., 5 (2015), pp. 748-760.

[30] H. B. ChE, L. Qi AND Y. Song, Column sufficient tensors and tensor complementarity problems, Front. Math. Chian, 13 (2018), pp. 255-276.

[31] Y. J. WANG, Convergence analysis of a block improvement method for polynomial optimization over unit spheres, Numer. Linear Algebra. Appl., 22 (2015), pp. 1059-1076.

[32] B. Qu AND H. X. ChAng, Remark on the successive projection algorithm for the multiple-sets split feasibility problem, Numer. Funct. Anal. Optim., 38 (2017), pp. 1614-1623.

[33] H. T. Che, Y. J. WANG And M. X. LI, A smoothing inexact Newton method for P-O nonlinear complementarity problem, Front. Math. China, 7 (2012), pp. 1043-1058. 